\title{
The use of double balloon enteroscopy for diagnosis and treatment of strictures of hepaticojejunal anastomoses after primary correction of bile duct injuries
}

\author{
Aliaksandr Varabei ${ }^{1,2}$, Yury Arlouski ${ }^{1,2}$, Egi Vizhinis ${ }^{1,2}$, Anatoli Shuleika $^{1,2}$, Natalia Lagodich $^{2,3}$, Natalia Derkacheva $^{3}$ \\ ${ }^{1}$ Belarusian Medical Academy of Postgraduate Education, Minsk, Belarus \\ ${ }^{2}$ Republican Centre of Surgical Gastroenterology, Coloproctology and Laser Surgery, Minsk, Belarus \\ ${ }^{3}$ Minsk Regional Clinical Hospital, Minsk, Belarus
}

Videosurgery Miniinv 2014; 9 (2): 219-225

DOI: $10.5114 /$ wiitm.2014.41635

\begin{abstract}
Introduction: Development of strictures of hepaticojejunal anastomoses (HJA) is observed in 6-30\% of patients and mortality after repeated reconstructive interventions ranges from $13 \%$ to $25 \%$. Double balloon enteroscopy (DBE) allows one to visualize the zone of Roux-en-Y anastomosis after reconstructive operations on the bile ducts for differentiation between stricture of HJA and recurrent cholangitis.

Aim: Report on the first experience of DBE of jejunal loop studies after reconstructive operations on the biliary tract. Material and methods: During the period 2002-2012 we performed in our centre 86 hepaticojejunostomies after iatrogenic bile duct injuries. Mean age was $51 \pm 6$ years. Patients with Roux-en-Y HJA and jejunum loop with Braun's bypass anastomosis who underwent DBE with endoscopic retrograde cholangiography (DBE-RChG) in our unit between February 2009 and December 2012 were enrolled in this study. A total of 33 procedures were performed during this period. All of them involved examination of HJA through a jejunum loop by DBE with capture of bile for bacteriology, Roux loop wall for biopsy and miniinvasive procedures.

Results: The DBE-RChG after visualization of the HJA zone was performed in 21 cases: 3 of them had the jejunum loop to Braun's bypass, 18 - HJA on the Roux loop. In 13 cases stricture of HJA was confirmed: at 6 reoperations were performed, in 7 - miniinvasive procedures (3 - laser vaporizations, 2 - stone extraction, 1 -lithotripsy, 1 - at the first stage stone extraction was carried out, then laser vaporization). The DBE-RChG was performed in 13 (61.9\%) patients. The overall diagnostic success with Braun's bypass was 100\%, after Roux-en-Y reconstruction in 10 of 13 cases (55.6\%). In connection with accumulation of experience, in 2012 diagnostic success in DBE-RChG of HJA on Roux loop increased to $81.3 \%$.

Conclusions: The MRI-ChG in our series frequently (10.3\%) shows a false-positive result in favor of HJA strictures. The DBE examination of HJA with additional cholangiography is a modern and precise method of detection of HJA strictures. Their DBE-balloon dilation and argon-laser vaporization or DBE lithoextraction are new ways of miniinvasive treatment.
\end{abstract}

Key words: double balloon enteroscopy, stricture, hepaticojejunal anastomosis. 


\section{Introduction}

Bile duct injury (BDI) is a severe complication that may have a great impact on the patient's physical and mental quality of life. The rate of iatrogenic BDI sustained during laparoscopic cholecystectomy is usually as high as $0.3-3 \%$, while for open cholecystectomy the rate has remained at $0.1-1 \%$ [1-3].

Bile duct injury following cholecystectomy is an iatrogenic catastrophe associated with significant perioperative morbidity and mortality, reducing longterm survival and quality of life, and high rates of subsequent litigation. Apparently, it is a great surgical challenge to handle biliary restructure after Roux-en-Y hepaticojejunostomy after bile duct injury. The operation can be much more difficult when compared with the first attempt of bile duct injury repair.

Development of hepaticojejunal anastomosis (HJA) stricture is observed in $6-30 \%$ of patients and mortality after repeated reconstructive interventions ranges from $13 \%$ to $25 \%[4,5]$.

Double balloon enteroscopy (DBE) is a new method of investigation of small bowel pathology. This technique allows one to visualize the zone of Rouxen-Y anastomosis after reconstructive procedures on the bile ducts for differential diagnosis between stricture of HJA and recurrent cholangitis.

\section{Aim}

Here we report on our experience with DBE for diagnosis and therapeutic interventions for strictures of Roux-en-Y HJA performed after iatrogenic BDI.

\section{Material and methods}

During the period 2002-2012 we performed in the Republican Centre of Surgical Gastroenterology, Coloproctology and Laser Surgery 86 hepaticojejunostomies with Roux-en-Y anastomosis after iatrogenic bile duct injuries and complications of their primary corrections. We collected those patients from all regions of our country.

Mean age was $51.7 \pm 6$ years (range: $21-68$ ). There were 49 cases of trauma and strictures of bile ducts and 37 strictures of previously performed HJA.

Bile duct injuries were all caused by cholecystectomy in other hospitals, including 47 cases with laparoscopic cholecystectomy, 28 - open cholecystectomy, 4 - procedures on the stomach and duodenum, 3 - abdominal trauma, 2 - liver resection, 1 - right hemicolectomy and 1 case - external drainage due to acute pancreatitis.

We classified bile duct injuries according to Bismuth. Type I injury was found in 9 patients, type II injury - in 22 , type III - in 37 , type IV - in 10 , type $\mathrm{V}-$ in 8 cases.

Eighty-six patients were operated on before admission to our centre: 52 - primary HJA with Roux-en-Y anastomosis; 3 - with Braun's bypass; 3 - choledochoduodenoanastomosis; 21 - external drainage of damaged bile ducts; 7 - primarily in our centre.

Patients with Roux-en-Y HJA and jejunum loop with Braun bypass who underwent DBE with endoscopic retrograde cholangiography (DBE-RChG) in our unit between February 2009 and December 2012 were enrolled in this study. A total of 33 procedures were performed during this period. DBE-RChG was carried out on 21 patients with full success in 13 cases (61.9\%).

Double balloon enteroscopy was performed with a pediatric enteroscope (EN-450P, Fujinon, Japan) with a working channel of $2.2 \mathrm{~mm}$ diameter and working length of $200 \mathrm{~cm}$. An ERC catheter $(250 \mathrm{~cm}, 1.8 \mathrm{~mm}$, Cook Medical, USA) was used for cannulation of the HJA. Vaporizations of the HJA strictures were performed with a National Three-Wave Laser (MUL-Surgeon, Belarus) with light guide $0.4 \mathrm{~mm}$ diameter.

Patient's preparation included a fasting period of at least $8 \mathrm{~h}$ before the procedure. Analgosedation was performed by administration of diazepam $0.5 \%$ and fentanyl $0.005 \%$ intravenously in a dosage as needed for sedation.

The enteroscope was sequentially pushed through the esophagus, stomach, duodenum, initial part of the jejunum, and further through Braun's bypass or end-to-side entero-enteroanastomosis into the jejunum Roux loop. A visual inspection of the zone of biliodigestive anastomosis was performed, as well as cannulation for staining of intrahepatic bile ducts, and additionally minimally invasive intervention was carried out. According to the indications stone extraction and laser vaporization of strictures of HJA were performed.

\section{Results}

Double balloon enteroscopy was performed on 33 patients ( 8 men; 25 women; mean age: 53.7 (range: 38-69) years with Roux-en-Y and Braun's 
bypass HJA. Indications for DBE-RChG included jaundice, biliary cholestasis, and cholangiolithiasis. This procedure was also used for differential diagnosis between strictures of HJA and recurrent cholangitis.

Initially, at different times after the operations performed before admission to our centre, MRI-ChG was carried out in 78 cases. It was impossible to delete strictures of HJA for 8 patients (10.3\%). In 1 case MRI-ChG was not performed because the patient had a femoral-head prosthesis.

The DBE-RChG procedures after visualization of the HJA zone were performed in 21 cases: 3 of them had the jejunum loop with Braun's bypass, 18 HJA on the Roux loop. Anastomosis functioned adequately in 8 patients with bile flow, the diameter remained wide, which allowed exclusion of anastomotic stricture, which was previously diagnosed on MRI-ChG. In 1 case, a patient with suspected stricture of HJA, in whom MRI was contraindicated, did not have a stricture. A sample of bile from the loop for bacteriological examination was taken from some patients. In 13 cases stricture of HJA was confirmed: in 6 reoperations were performed, in 7 cases - minimally invasive procedures ( 3 - laser vaporizations, 2 - stone extraction, 1 - at the first stage stone extraction was carried out, then laser vaporization, 1 - laser lithotripsy). The ERC was performed in $12(57.2 \%)$ patients, DBE-RChG was successful in 13 cases, including 10 - in HJA on the Roux loop (55.6\%) and in 3 cases with Braun bypass (100\%). Overall diagnostic success for HJA on the Roux loop and loop with Braun bypass was $61.9 \%$. In connection with accumulation of experience, in 2012 diag-

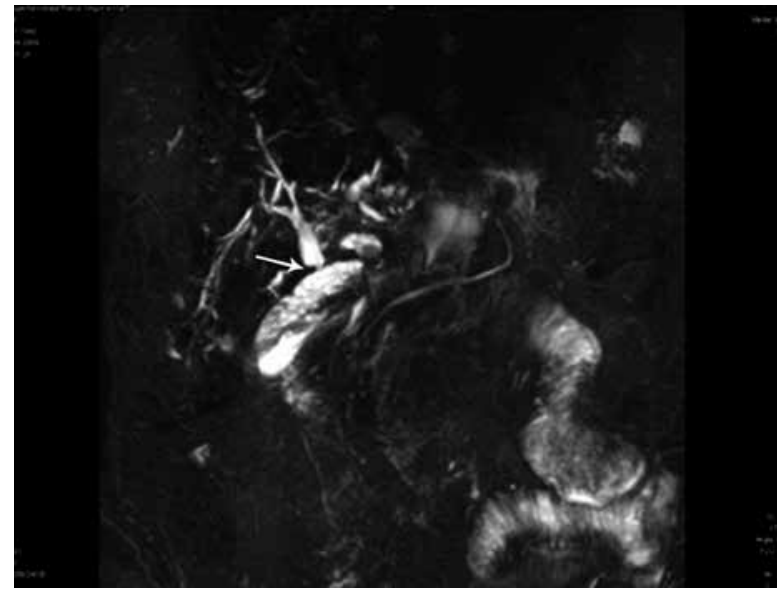

Photo 1. The MRI-ChG: stricture of HJA (shown by arrow)

nostic success in DBE-RChG of HJA on the Roux loop increased to $81.3 \%$.

\section{Case 1}

Patient S.R. had intermittent fever and persistent epigastric abdominal pain for 1 month. Nine years earlier, she underwent HJA with Braun's bypass because of an iatrogenic injury. An MRI-ChG revealed stricture of HJA (Photo 1).

As the next step we performed DBE. When an area of the HJA was shown stricture was excluded: it was a wide anastomosis with bile flow (Photos $2 \mathrm{~A}$ and $2 \mathrm{~B}$ ), which allowed us to establish the diagnosis of recurrent cholangitis and administer conservative therapy.

This observation underlines the importance of DBE for the differential diagnosis, even in the case of

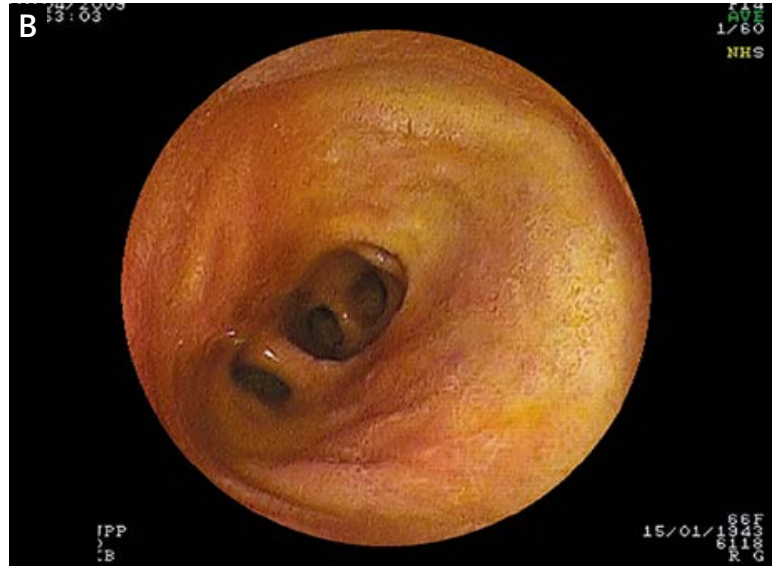

Photo 2. Double balloon enteroscopy: A - wide anastomosis, stricture excluded, B - enteroscope introduced above HJA 

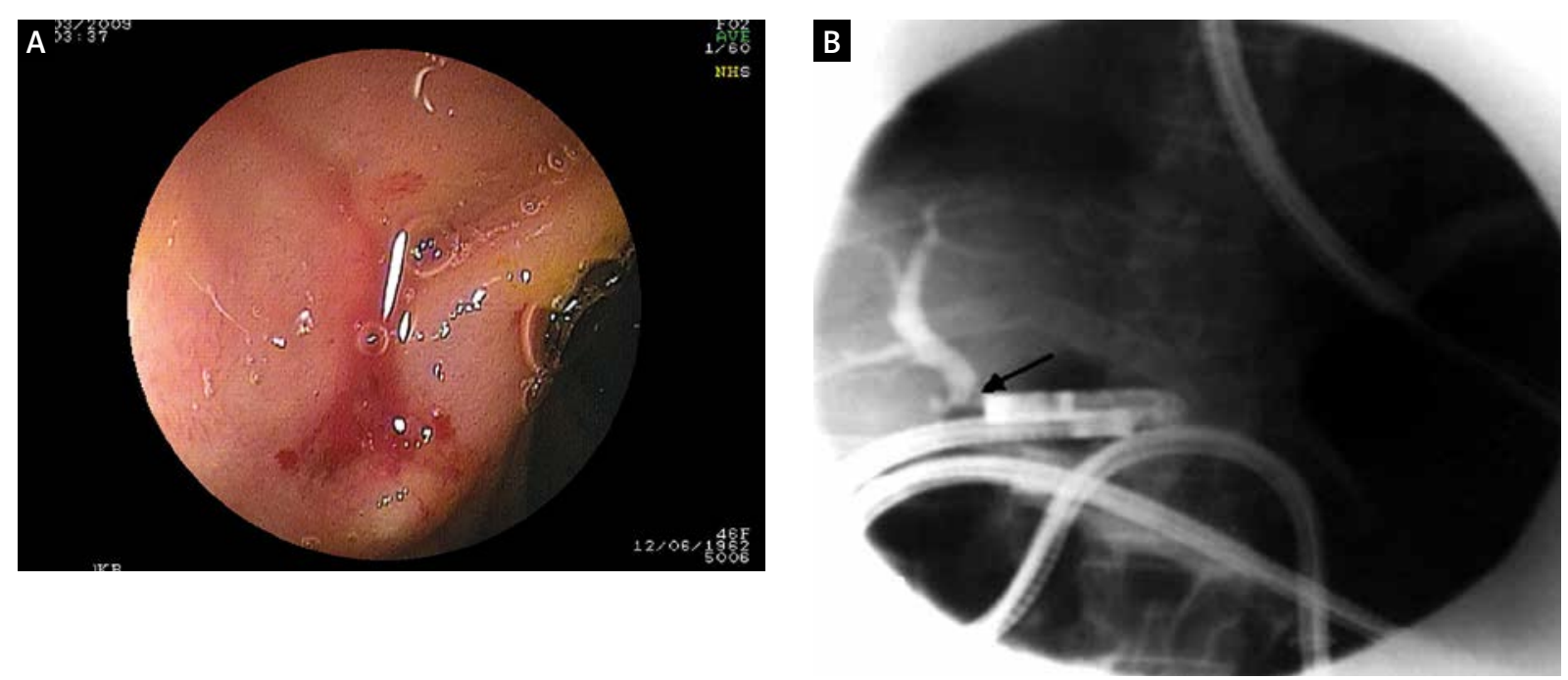

Photo 3. Double balloon enteroscopy: A - scar-restricted HJA can be seen, B - fluoroscopic picture of ERChG through DBE (stricture in distal part of right hepatic duct, bile duct stone - shown by arrow)

previously diagnosed stricture of HJA on the results of an MRI-ChG.

In another case, a stricture biliodigestive anastomosis with the right hepatic duct to a Braun's bypass was confirmed. It was possible to perform cannulation of the scar-restricted anastomosis and to stain intrahepatic bile ducts (Photos $3 \mathrm{~A}$ and $3 \mathrm{~B}$ ).

In 3 cases in 2010 after visualization of the HJA stricture laser recanalization was performed using the National Three-Wave Laser "MUL-Surgeon". The diameter of the anastomosis with severe scarring in these observations ranged from $2.5 \mathrm{~mm}$ to $5 \mathrm{~mm}$. All patients had recurrent cholangitis.

\section{Case 2}

Patient V.B. had jaundice and epigastric abdominal pain for 2 weeks. One year earlier, she underwent HJA with Roux-en-Y reconstruction on the hepatic duct because of an iatrogenic injury. An MRI-ChG revealed stricture of HJA not far from the confluence zone. We performed DBE and when the area of HJA was shown stricture was confirmed: scar-restricted HJA in the form of two openings above the confluence was visualized (Photo $4 \mathrm{~A}$ ). We managed to contrast both hepatic ducts separately (Photo $4 \mathrm{~B}$ ).

In this case laser vaporization of stricture of HJA was performed with a light guide wavelength of $1420 \mathrm{~nm}$ and power 10 watts in several stages (Photo 5 A). During 1 week control of inspection of zone of HJA was performed, where, after vaporiza- tion, sufficient width anastomosis with adequate bile flow was obtained (Photo $5 \mathrm{~B}$ ).

In 1 case, bile duct stone formed above the stricture. The stone extraction was performed with an endoscopic basket (Photos $6 \mathrm{~A}$ and 6 B). At control cholangiography proper functioning biliodigestive anastomosis without rendering concrement was observed.

The procedure could not be achieved in 7 patients in whom the scope was unable to reach the afferent loop due to sharp angulation of jejunojejunostomy end-to-side anastomosis. We performed bile extraction and biopsy of the wall of the jejunum loop for bacteriological examination. In 1 case according to the bacteriological seeding candidosis cholangitis was diagnosed. In 6 patients in the presence of intact bile in the distal loop of Roux pathology from biliodigestive anastomosis was eliminated.

The overall diagnostic success with Braun's bypass was $100 \%$, after Roux-en-Y reconstruction $55.6 \%$. Therapeutic success was 53.9\%. However, in connection with accumulation of experience, in 2012 diagnostic success was increased to $81.3 \%$.

The procedure lasted a mean time of 85 (range: 45-120) min (with cannulation HJA - 120 min).

\section{Discussion}

The first successful endoscopy of parts of the small bowel was executed by Hiratsuka in 1971 using an endoscope probe [6]. However, this method of visualization was not widespread due to insufficient 

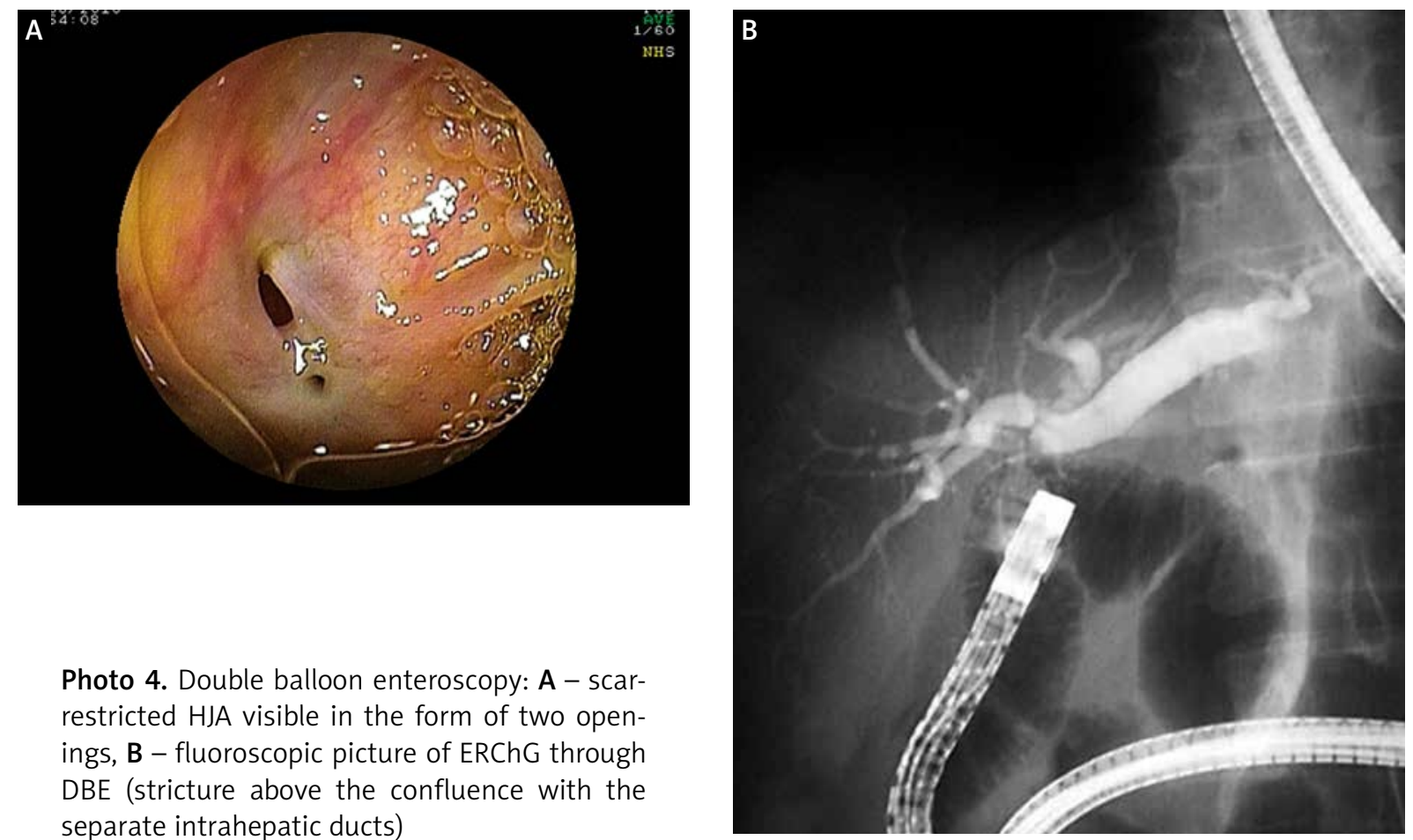

Photo 4. Double balloon enteroscopy: A - scarrestricted $\mathrm{HJA}$ visible in the form of two openings, $\mathbf{B}$ - fluoroscopic picture of ERChG through DBE (stricture above the confluence with the separate intrahepatic ducts)
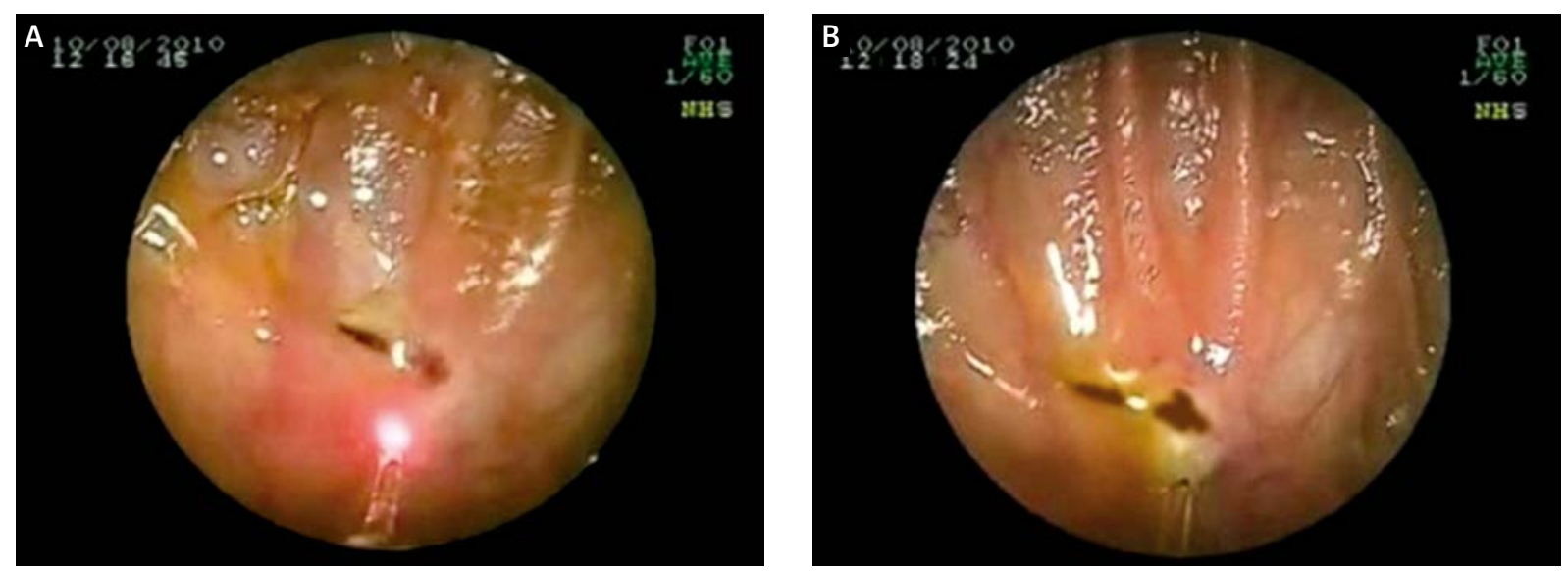

Photo 5. Double balloon enteroscopy: A - laser vaporization of stricture of HJA, B - controlling inspection of zone of HJA

maneuverability, duration of the procedure $(6-8 \mathrm{~h})$, absence of air insufflation possibility and also because of numerous complications.

The beginning of DBE creation is associated with the introduction of Push-endoscopy by Yamamoto in 1997, performed on a patient with a small intestine hemangioma [7].

In 2000 development of DBE in cooperation with the company Fujinon began and in 2001 serial production of the enteroscope started [8]. Yamamoto and Sugano published the first article about the new method of research in 2003 [9]. A number of publications have been available lately which concern carrying out small intestine endoscopy with the altered anatomy after reconstructive operations on the bile duct (including OLT), stomach and pancreas: Roux-en-Y HJA, Billroth-II stomach resection, Whipple procedure $[10,11]$. During this period (according to Medline and The Cochrane Library) about 20 publications have appeared, informing about 129 patients on whom the authors performed DBE after various HJA (Table I). 

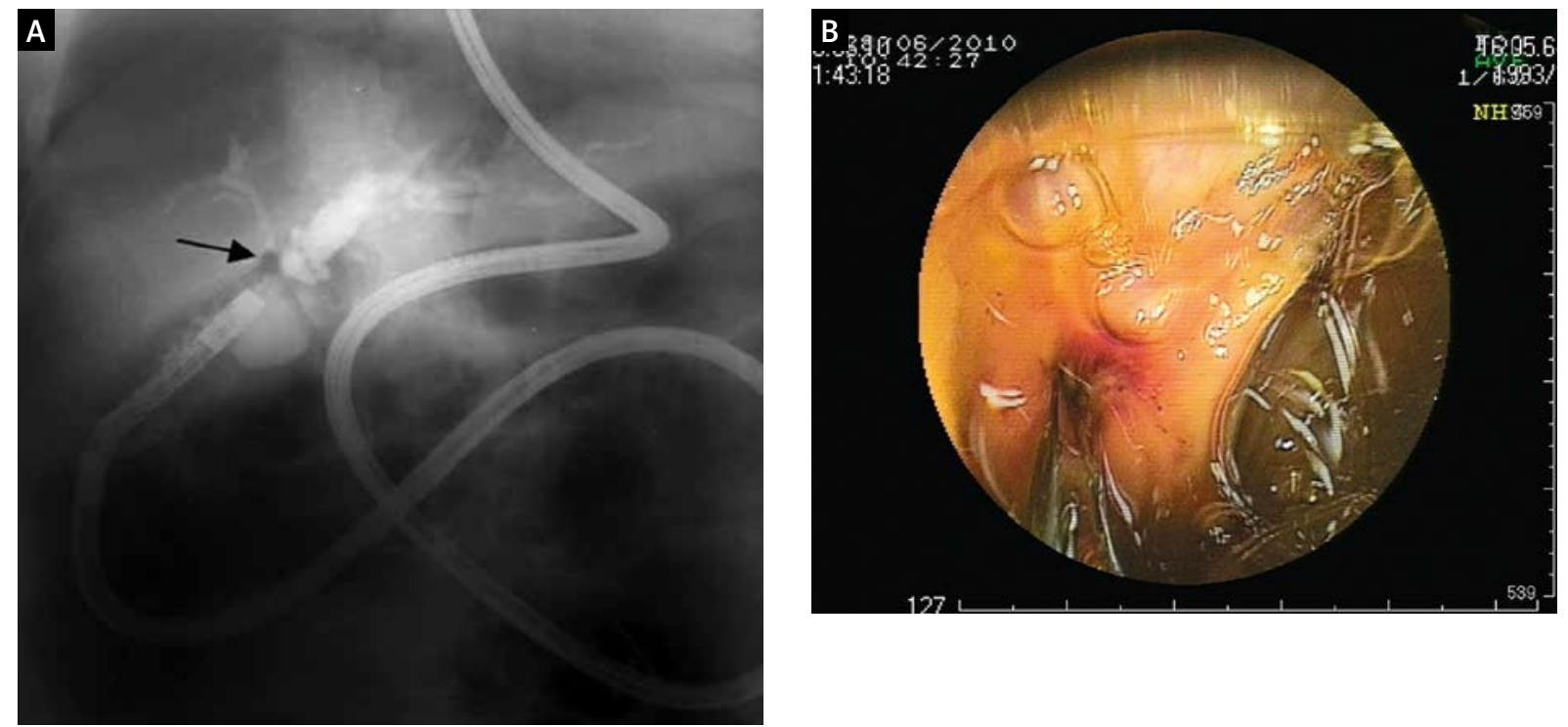

Photo 6. Double balloon enteroscopy: A - fluoroscopic picture of ERChG through DBE (bile duct stone formed above the stricture - shown by arrow), B - stone extraction by endoscopic basket through HJA

Table I. Meta-analysis of DBE-ERChG in Rouxen-Y anastomosis

\begin{tabular}{|lcc|}
\hline Author, year [reference] & $\begin{array}{c}\text { Number } \\
\text { of cases }\end{array}$ & $\begin{array}{c}\text { Diagnostic } \\
\text { success (\%) }\end{array}$ \\
\hline Aabakken et al., 2007 [12] & 13 & 92.3 \\
\hline Chu et al., 2008 [13] & 5 & 60 \\
\hline Koornstra, 2008 [14] & 3 & 100 \\
\hline Maaser et al., 2008 [15] & 11 & 100 \\
\hline Parlak et al., 2009 [16] & 14 & 92.9 \\
\hline Moenkemueller et al., 2010 [17] & 32 & 82 \\
\hline Hulagu et al., 2010 [18] & 10 & 80 \\
\hline Raithel M et al., 2010 [19] & 24 & 87.5 \\
\hline Our series, 2012 & 21 & $55.6^{\star}\left(81.3^{* *}\right)$ \\
\hline
\end{tabular}

${ }^{\star}$ Overall DBE-RChG diagnostic success (2009-2012), ${ }^{\star \star} D B E-R C h G$ success only in 2012

This report illustrated the utility of DBE to perform diagnostic and therapeutic ERChG in patients after HJA to whom repair of the injury was done due to cholecystectomy. In our series the success rate to reach anastomosis was $55.6 \%$. In connection with accumulation of experience, in 2012 the diagnostic success in DBE-RChG of HJA on the Roux loop increased to $81.3 \%$.

According to the literature, survey of the jejunum Roux loop is reached in $90-93 \%$ of cases, and the success of carrying out ERChG through DBE and survey of the HJA zone varies from $60 \%$ to $94.4 \%$ [1215]. The risk of complications after ERChG does not exceed $5 \%$, which is lower than during performance of percutaneous interventions on the common bile duct [14]. We found that in 7 cases the anastomosis was not recognized during insertion of the DBE. Perhaps it could be explained by the angulated location of the afferent limb opening at the jejunal anastomosis.

The localization of the HJA was somewhat variable, but it was generally located in the lower left part of the endoscopic view. Generally, the opening of the HJA was very small. Therefore, it is important to inspect the blind pouch carefully and search for the HJA opening, which usually is located $10-15 \mathrm{~cm}$ distal from the blind ending.

At the moment of carrying out the given research there is a possibility to perform balloon dilatation, stenting, laser vaporization, staining of intrahepatic bile ducts, stone extraction, and also biopsy of HJA zones $[14,16,20]$. In our case series, in 3 patients we performed laser vaporization of stricture of HJA, stone extraction from intrahepatic bile duct - 1 , and cleaning putty from the bile duct.

According to the data of some authors, during the research a number of complications (from $0.8 \%$ to $4.3 \%$ ) deserve the greatest attention: bowel puncture, abdominal pain, bleeding, fever and, in some situations, development of acute pancreatitis [14]. To avoid puncture of the small intestine, some techniques are useful: the procedure is recommended to be carried out under the strict control of fluoroscopy; 
for adequate passage of the zone of jejuno-jejuno end-to-side anastomosis it is necessary to straighten partially a corner of the anastomosis, using an external tube enteroscope with its subsequent accurate advancement in the Roux loop.

Magnetic resonance imaging-cholangiography gives false-positive data in favor of stricture of HJA. Sensitivity and specificity of MRI in our study were $84.4 \%$ and $63 \%$, DBE with ERChG $-96.1 \%$ and $93.4 \%$ respectively. The advent of DBE provided new possibilities for a visual estimation of functioning of biliodigestive anastomoses, performance of ERChG and adequate authentic interpretation of the diagnostic data [21].

\section{Conclusions}

The DBE-ERChG is a useful tool for diagnosis and therapeutic procedures in patients with Roux-en-Y and Braun's bypass-altered anatomy. Diagnostic efficiency of DBE visualization of HJA depends on the endoscopist's experience, features of formation of jejunojejunal end-to-side anastomosis, and presence of adhesions in the abdominal cavity. The MRI-ChG in our series shows a false-positive result in $10.3 \%$ of cases in favor of HJA strictures. Laser vaporization of stricture of HJA is a new, effective and miniinvasive procedure for the decrease of biliary hypertension.

\section{References}

1. Melton GB, Lillemoe KD, Cameron JL, et al. Major bile duct injuries associated with laparoscopic cholecystectomy: effect of surgical repair on quality of life. Ann Surg 2002; 235: 888-95.

2. Dolan JP, Diggs BS, Sheppard BC, Hunter JG. Ten-year trend in the national volume of bile duct injuries requiring operative repair. Surg Endosc 2005; 19: 967-73.

3. Kaklamanos IG, Birbas KN, Bonatsos GN. Iatrogenic injury of the extrahepatic bile ducts. Surgical reconstruction. In: Liver and Biliary tract surgery. Embryological anatomy to 3D-imaging and transplant innovations. Karaliotas CCh, Broelsh CE, Habib NA (eds.). Springer Wien, New York 2006; 179-91.

4. Schmidt SC, Langrehr JM, Hintze RE, et al. Long-term results and risk factors influencing outcome of major bile duct injuries following cholecystectomy. Br J Surg 2004; 92: 76-82.

5. Walsh RM, Henderson JM, Vogt DP, Brown N. Long-term out come of biliary reconstruction for bile duct injuries from laparoscopic cholecystectomies. Surgery 2007; 142: 450-57.

6. Hiratsuka H. Endoscopic diagnosis in the small intestine. Stom ach Intest 1972; 7: 1679-85.

7. Mulder CJJ. Atlas of double balloon endoscopy. In: History and development of double balloon endoscopy. Medconnect $\mathrm{GmbH}$, Munich 2007; 2-4.
8. Sugano K, Yamamoto H, Kita H. Double balloon endoscopy: theory and practice. Springer 2006; 9-11.

9. Yamamoto H, Sugano K. A new method of enteroscopy - the double balloon method. Can J Gastroenterol 2003; 17: 273-4.

10. Fahndrich M, Saudmann M, Heike M. A facilitated method for endoscopic interventions at the bile duct after Roux-en-Y reconstruction using double balloon enteroscopy. Z Gastroenterol 2008; 46: 335-8.

11. Koornstra JJ, Fry L, Monkemuller K. ERCP with balloon-assisted enteroscopy technique: a systematic review. Dig Dis 2008; 26: 324-9.

12. Aabakken L, Bretthauer M, Line PD. Double-balloon enteroscopy for endoscopic retrograde cholangiography in patients with a Roux-en-Y anastomosis. Endoscopy 2007; 39: 1068-71.

13. Chu YC, Yang CC, Yen YH, et al. Double-balloon enteroscopy application in biliary tract disease - its therapeutic and diagnosis function. Gastrointest Endosc 2008; 68: 585-91.

14. Koornstra JJ. Double balloon enteroscopy for endoscopic retrograde cholangiopancreaticography after Roux-en-Y reconstruction: case series and review of the literature. Neth J Med 2008; 66; $275-9$.

15. Maaser C, Lenze F, Bokemeyer M, et al. Double balloon enteroscopy: a useful tool for diagnostic and therapeutic procedures in the pancreaticobilliary system. Am J Gastroenterol 2008; 103: 3218-9.

16. Parlak E, Cicek B, Disibeyaz S, et al. Endoscopic retrograde cholangiography by double balloon endoscopy in patients with Roux-en-Y hepaticojejunostomy. Surg Endosc 2009; 6: 45-7.

17. Moenkemueller K, Sancar A, Zabielski M, et al. ERCP with the double balloon-assisted enteroscope technique in patients with biliary disorders and postsurgical Roux-en-Y anastomosis - a bicenter systematic prospective observational study. $1^{\text {st }}$ International Conference on Capsule Endoscopy and Double Balloon Endoscopy, Paris, France, 27-28 August 2010; P61.

18. Hulagu S, Senturk O, Celebi A, et al. Endoscopic retrograde cholangiography by double-balloon enteroscopy: indications and therapeutic yield. $1^{\text {st }}$ International Conference on Capsule Endoscopy and Double Balloon Endoscopy, Paris, France, 27-28 August 2010; P33.

19. Raithel M, Nagel A, Dormann H, et al. Modern enteroscopic interventions and characterization of non-malignant post-surgical biliary anastomosis by double balloon endoscopy. $1^{\text {st }} \mathrm{In}$ ternational Conference on Capsule Endoscopy and Double Balloon Endoscopy, Paris, France, 27-28 August 2010, P41.

20. Bruno M. Double balloon scope for endoscopic retrograde cholangiopancreatography. J Med 2008; 66: 267-8.

Received: 21.01.2014, accepted: 17.02.2014. 